\title{
Análise de Redes Neurais Convolucionais e Técnicas de Pré-processamento para Identificação de Dentes Serotinos com Cistos
}

\author{
Danilo Alves Oliveira ${ }^{1}$, Joniel Bastos Barreto ${ }^{1}$, Iago Magalhães de Mesquita ${ }^{2}$, Iális \\ Cavalcante de Paula Júnior ${ }^{1}$, Filipe Nobre Chaves ${ }^{3}$, Marcelo Bonifácio da Silva \\ Sampieri $^{3}$
}

${ }^{1}$ Programa de Pós-Graduação em Engenharia Elétrica e da Computação - Universidade Federal do Ceará (UFC) - Sobral - CE - Brasil

${ }^{2}$ Eixo de Controle e Processos Industriais - Instituto Federal de Educação, Ciência e Tecnologia do Estado do Ceará (IFCE) - Sobral - CE - Brasil.

${ }^{3}$ Curso de Graduação em Odontologia - Universidade Federal do Ceará. (UFC) - Sobral $-\mathrm{CE}-$ Brasil.

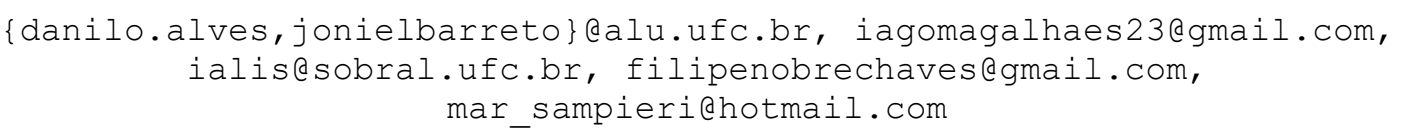

\begin{abstract}
The extraction of third molars is always under debate among dentists, because pathologies can arise with their permanence. Aiming to help diagnose this paper seeks to automate the detection of third molars with cysts in radiography images. For this, two architectures of Convolutional Neural Networks (CNN) are analyzed for classification and experimented with image preprocessing techniques. One of these proposals, using morphological contrast, obtained better performance, with emphasis on the precision of 0.93 and F1-score of 0.84. The results show that the proposal allows automation in the diagnosis of cysts.
\end{abstract}

Resumo. A extração dos terceiros molares está sempre em debate entre dentistas, isto porque podem surgir patologias com a sua permanência. Visando auxiliar no diagnóstico o presente trabalho procura automatizar a detecção de terceiros molares com cistos em imagens de radiografias. Para isso, são analisadas duas arquiteturas de Redes Neurais Convolucionais (CNN) para a classificação e experimentadas com técnicas de pré-processamento de imagem. Uma destas propostas, com uso de contraste morfológico, obteve melhor performance, com destaque à precisão de 0,93 e Fl-score de 0,84. Os resultados demonstram que a proposta permite automatização no diagnóstico de cistos.

\section{Introdução}

Diversas alterações patológicas podem surgir com a permanência dos terceiros molares, uma delas é o aparecimento de cistos odontológicos [Zanetti et al. 2009]. Um cisto dental é clinicamente definido como uma camada revestida de tecido epitelial, como um saco ou bolsa, em que se acumula um líquido preenchido de células mortas e bactérias. Em 
[Slootweg 1987] é apontado que patologias como cisto ou tumor em relação a um dente em desenvolvimento são uma das três principais causas de desvio do padrão normal de erupção do dente.

Um dos cistos mais comuns é o cisto dentígero, que se desenvolve dentro de um folículo dentário nas proximidades ou ao redor de um dente não erupcionado, comumente encontrados em torno dos terceiros molares [Ulaganathan et al. 2012], estes dentes também são conhecidos como dentes sisos ou dentes serotinos. Em um estudo de [Salehinejad et al.2013] sobre a ocorrência de cisto odontogênico glandular de mandíbula associado a serotino retido, obteve que esta patologia comum nesse dente pode ser diagnosticada radiograficamente como cisto dentígero. Além disso, esses dentes podem apresentar outros problemas como aponta uma pesquisa realizada por [Patil 2013], na qual obteve que $69,7 \%$ dos terceiros molares impactados na população indiana ocidental com um espaço folicular radiograficamente normal estavam associados a patologias quando submetidos a exame histológico.

A maioria dos cistos possuem um crescimento inicial lento e sem dor, o que dificulta o seu diagnóstico, e em muitos casos passam despercebidos. Determinar a localização e o tamanho de doenças dentais mesmo com a presença de indicadores físicos como dor, cor, e perda de função é uma tarefa desafiadora e ainda mais difícil em casos assintomáticos. Em tais circunstâncias, o uso de radiografias dentárias com a interpretação de um profissional bem treinado se faz necessário para o diagnóstico. Contudo, considerando o fator humano, erros que levariam a um tratamento incorreto devido a um diagnóstico errado podem ser cometidos mesmo por especialistas [Birdal et al. 2016]. Diante disso, pesquisas estão sendo desenvolvidas envolvendo Visão Computacional (VC) e Inteligência Artificial (IA) para análise de imagens odontológicas visando otimizar o processo de diagnóstico.

Muitas das aplicações envolvendo VC e IA consistem na classificação de padrões, em que apresentado um padrão para o algoritmo é possível identificá-lo em outras imagens. Aplicadas a análise de imagens odontológicas é utilizada em problemas como selecionar patologias em radiografias, separar o cortical mandibular, entre outros. Essas aplicações auxiliam o profissional de saúde no diagnóstico clínico e possibilitam otimizar e elevar a qualidade dos diagnósticos, consequentemente o tratamento dos pacientes.

Em vista disso, o presente trabalho visa apresentar uma metodologia utilizando Redes Neurais Convolucionais (do inglês Convolutional Neural Networks - CNN) para identificar a presença de cistos em imagens de terceiros molares, investigando a influência de técnicas de pré-processamento. Essa metodologia desenvolvida é uma etapa que compõe uma pesquisa voltada para a segmentação de dentes sisos em imagens de radiografias panorâmicas e classificação da presença de cistos, por meio de aprendizagem profunda.

\section{Trabalhos relacionados}

$\mathrm{Na}$ literatura, encontram-se diversas revisões com pesquisas voltadas para o auxílio de diagnósticos em casos odontológicos, tanto para análise de patologias quanto para o desenvolvimento dos dentes sisos.

Em [Banar et al. 2020] foi desenvolvido um estudo voltado para automatizar o processo de estadiamento dos terceiros molares usando CNNs. Foi utilizando um conjunto de 400 radiografias panorâmicas, sendo 20 para cada estágio de 
desenvolvimento por sexo, encenadas em consenso entre três observadores. Os resultados apresentaram uma segmentação dos terceiros molares com uma média Dice em 93\% e classificação dos estágios com precisão de $54 \%$, erro absoluto médio de 0,69 estágios e coeficiente kappa de Cohen ponderado linear de 0,79.

Em [Divya et al. 2017] foram apresentados dois algoritmos distintos de processamento de imagens para realizar uma caracterização de patologias dentais por meio de imagens digitais de raios-X panorâmicas. Na primeira abordagem é realizada a detecção de cárie dentária usando transformação negativa hibridizada. $\mathrm{Na}$ segunda abordagem é feita a análise estatística da textura das imagens dentais contendo cistos e cáries. Correlacionando os resultados obtidos com o diagnóstico feito pelas radiologias maxilofaciais ambos os métodos se mostraram satisfatórios.

Em [Lee, Kim e Jeong et al. 2020] foi utilizado uma CNN com arquitetura GoogLeNet Inception - v3, para a detecção de lesões císticas odontogênicas (OCLs) e avaliaram o diagnóstico de três tipos de lesões - queratocistos odontogênicos, cistos dentígeros e cistos periapicais usando radiografia panorâmica e imagens tomográficas computadorizadas de feixe cônico (CBCT). O trabalho desenvolvido demonstrou que os três tipos de OCLs, são efetivamente detectados e diagnosticados com base na arquitetura CNN profunda. O modelo pré-treinado apresentou melhor desempenho para o conjunto de imagem CBCT, com valores de acurácia $=91,4 \%$, sensibilidade $=96,1 \%$ e especificidade $=77,1 \%$, do que por outros modelos usando imagens panorâmicas, com acurácia $=0,847$, sensibilidade $=88,2 \%$ e especificidade $=77,0 \%$.

Em [Devi et al. 2020] foi proposto um novo limiar baseado em região, denominado Regiões Extremamente Estáveis Maximamente (MSER), para realizar a extração de cisto dentário em imagens digitais de raios- $X$ dentais utilizando um novo limiar baseado em região. $\mathrm{O}$ método proposto mostrou-se muito eficiente e preciso na extração do limite cístico dentário. É possível observar com base nos resultados da segmentação do cisto baseada no MSER em radiografias odontológicas que existe uma correlação significativa encontrada entre a região cística definida pelos profissionais especialistas.

Em [Kwon et al. 2020] foi desenvolvido um novo framework de CNN, modificado de $Y O L O$ v3, com aumento de dados para detecção e classificação de múltiplas doenças para automatizar o diagnóstico de cistos e tumores odontogênicos de ambas as mandíbulas em radiografias panorâmicas. O conjunto de dados é formado por 1282 radiografias, sendo 350 cistos dentígeros (DCs), 302 cistos periapicais (PCs), 300 ceratocistos odontogênicos (OKCs), 230 ameloblastomas (ABs) e 100 maxilares normais sem doença. Além disso, o conjunto foi aumentado 12 vezes por técnicas de transformação. O método desenvolvido apresentou um desempenho geral da classificação para as doenças usando a CNN com conjunto de dados não aumentado melhorado de $78,2 \%$ de sensibilidade, $93,9 \%$ de especificidade, $91,3 \%$ de precisão e $86 \%$ de acurácia para $88,9 \%, 97,2 \%, 95,6 \%$ e 0,94 , respectivamente, usando a CNN com conjunto de dados aumentado. 


\section{Materiais e métodos}

\subsection{Banco de imagens}

As imagens utilizadas foram obtidas através de uma parceria com o curso de Odontologia da UFC - Campus Sobral, nas quais os cistos foram marcados por dois professores especialistas na área. As imagens são de radiografias panorâmicas, com nível de cinza e codificação de 8 bits. Através dessas, foram extraídas manualmente sub-imagens dos dentes sisos das radiografias originais para formação da base utilizada. A base possui 243 imagens de dentes sisos, com 118 contendo cistos e 125 saudáveis. Exemplos de imagens do dataset são apresentados na Figura 1.

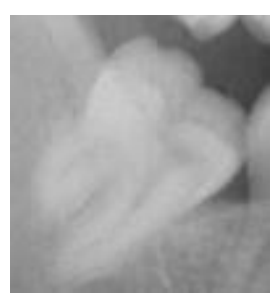

(a)

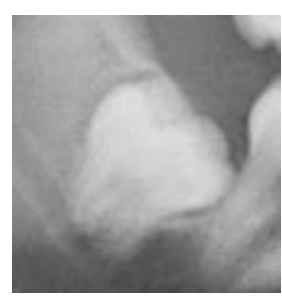

(b)

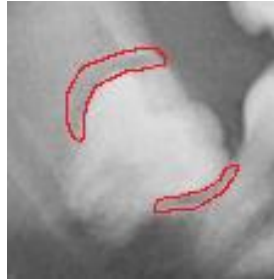

(c)

Figura 1. Imagens do banco. (a) imagem sem cisto. (b) imagem com cisto. (c) imagem com ênfase no cisto.

A Figura 1(a) apresenta uma imagem com o dente serotino diagnosticado como saudável. A Figura 1(b) apresenta um exemplo com presença de cisto, nela é possível observar as regiões radiolúcidas indicando o cisto ao redor do dente. A Figura 1(c) indica em vermelho a região do cisto da Figura 1(b).

\subsection{Pré-processamento}

$\mathrm{O}$ pré-processamento é uma das etapas fundamentais da VC que tem como objetivo melhorar a imagem em aspectos relevantes em função do que será feito com ela, seja corrigindo defeitos oriundos da aquisição ou adaptando para o problema. Neste trabalho são comparadas duas técnicas de contraste: ajuste de contraste por intensidade dos pixels e operação morfológica.

Antes de executar as técnicas de ajuste do contraste é executado um filtro gaussiano nas imagens para que seja reduzido possíveis ruídos de alta frequência adquiridos na aquisição. O filtro gaussiano reduz os ruídos aplicando uma convolução da função gaussiana aos pixels, gerando uma nova imagem com um aspecto de suavização em relação à original. A função é dada por

$$
G(x, y)=\frac{1}{2 \pi \sigma^{2}} e^{-\frac{x^{2}+y^{2}}{2 \sigma^{2}}},
$$

sendo $\sigma$ o controle da largura de banda do filtro e $x$ e $y$ são os valores do eixo das abscissas e ordenadas, respectivamente.

A técnica de contraste por ajuste de tons mapeia os valores de intensidade da imagem considerando um intervalo dos níveis de intensidade dos pixels, os valores acima e abaixo desse intervalo são saturados gerando assim uma nova imagem com o contraste realçado. Para aplicar essa técnica é preciso definir os valores mínimo e máximo de um intervalo, e um valor para saturação máxima e mínima, podendo ser um valor de correção 
ou valores em que se deseja mapear os pixels. Assim sendo, todos os pixels abaixo do limite mínimo são considerados com o valor de saturação mínima e os acima do limite máximo com o valor de saturação máxima.

O contraste por operação morfológica realiza modificações no histograma das imagens através de operações de morfologia matemática, essa técnica é baseada no trabalho de [Birdal et al. 2016]. A operação é definida por

$$
I_{c}=\left(I+T_{\text {hat }}(I)\right)-B_{\text {hat }}(I),
$$

em que $I$ representa a imagem de entrada, $I_{c}$ a imagem realçada e $T_{\text {hat }}$ e $B_{\text {hat }}$ as transformadas morfológicas Top-hat e Bottom-hat.

\subsection{Redes Neurais Convolucionais}

As Redes Neurais Convolucionais são modelos de classificação baseados no processo biológico da visão, são considerados variações das redes perceptrons de múltipla camada. Essas redes substituem a multiplicação geral da matriz pela operação de convolução ao menos em uma camada [Goodfellow, Bengio e Courville 2016].

A CNN consegue aplicar filtros em imagens utilizando convoluções sem eliminar as relações de vizinhança. As camadas responsáveis por isso são chamadas de camadas convolucionais, elas possuem os neurônios organizados em mapas de características. Os mapas são obtidos através das saídas de janelas de neurônios que cobrem uma parte da imagem que tenha utilizado um filtro em comum [LeCun et al. 2015]. O passo de movimentação da convolução é chamado de strides e seu valor está relacionado à dimensão de saída do filtro. As CNNs também podem ser aplicadas com uma técnica conhecida como padding, que consiste em adicionar zeros nas bordas da imagem antes da convolução, a fim de manter as dimensões originais após o processo.

Em conjunto com as camadas convolucionais, a CNN possui a camada de pooling, que tem como objetivo reduzir a dimensão dos mapas de características e obter invariância ao deslocamento [Goodfellow, Bengio e Courville 2016]. Entre as camadas da rede, também é possível utilizar uma técnica conhecida como dropout, que retira aleatoriamente neurônios das camadas com o objetivo de evitar o sobreajuste dos pesos [Gu et al. 2015].

Após essas camadas de extração, a CNN possui uma ou mais camadas totalmente conectadas, geralmente feed-forward. Essas camadas são responsáveis por classificar os recursos vindos das camadas anteriores.

\subsection{Métricas estatísticas}

Os indicadores obtidos para avaliar os resultados são medidas utilizadas frequentemente na área da saúde. Estas são calculadas através da análise da resposta de um observador para uma determinada amostra com a resposta correta fornecida pelo especialista. Nessa pesquisa, as amostras são as imagens dos dentes sisos e os observadores são os algoritmos experimentos na metodologia.

As amostras selecionadas pelo algoritmo com presença de cistos e que também foram marcadas pelos especialistas com cisto, são agrupadas como Verdadeiros Positivos (VP). As que foram classificadas pelo algoritmo como cistos, mas que não constam como tal para os especialistas, são agrupadas como Falsos Positivos (FP). As que foram 
marcadas como saudáveis pelo algoritmo e que também correspondem às saudáveis pelos especialistas, são agrupadas como Verdadeiros Negativos (VN). Por fim, as amostras marcadas como saudáveis pelo algoritmo, mas que constam com presença de cisto pelos especialistas, são agrupadas como Falsos Negativos (FN).

Através desses grupos, são calculadas as métricas de acurácia, precisão, sensibilidade e F1-score representadas nas equações 3, 4, 5 e 6.

$$
\begin{aligned}
& \text { Acurácia }=\frac{V P+V N}{V P+V N+F P+F N} \\
& \text { Precisão }=\frac{V P}{V P+F P} \\
& \text { Sensibilidade }=\frac{V P}{V P+F N} \\
& F 1-\text { score }=\frac{2 * \text { Precisão } * \text { Sensibilidade }}{\text { Precisão+Sensibilidade }}
\end{aligned}
$$

A acurácia indica a performance geral da classificação, o quão bem o modelo classificou corretamente. A precisão indica o quão bem o modelo classificou corretamente amostras com cistos. A sensibilidade, ou recall, representa a proporção de marcações indicadas como positivas que estão corretas. A F1-score é um indicativo conjunto da precisão e sensibilidade que apresenta valor mais próximo dos menores valores que uma média comum.

\section{Método Proposto}

Para realizar a investigação proposta, os exames foram coletados, diagnosticados por especialistas e extraídas as imagens dos dentes sisos. As CNNs são aplicadas nessas imagens, sendo experimentadas com a presença e ausência da etapa de préprocessamento.

Dessa forma, a pesquisa consta com quatro etapas: Aquisição, pré-processamento, classificação e análise dos resultados. As etapas estão dispostas na Figura 2. Na etapa de aquisição, é realizada a extração das sub-imagens dos dentes sisos e a formação do banco. Em seguida, a metodologia pode seguir por dois caminhos: Aplicação direta das CNNs ou aplicar um pré-processamento previamente nas imagens. Após a classificação, os resultados são analisados para selecionar a melhor combinação da metodologia.

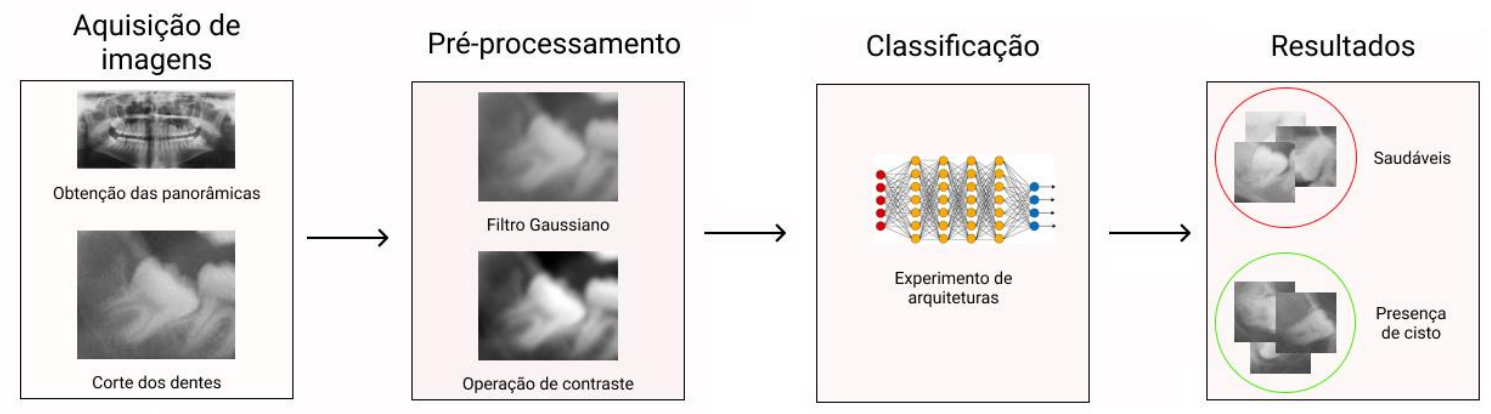

Figura 2. Metodologia proposta. 


\subsection{Pré-processamento das imagens}

O filtro de ruídos utilizado é o gaussiano com desvio padrão de 0,5 . Para as operações de contraste foram aplicadas duas técnicas: morfológico e ajuste das intensidades de cinza. No contraste morfológico é utilizado um elemento estruturante circular com tamanho 30 e no contraste por ajuste os valores de tons da imagem são mapeados para novos valores dentro do intervalo de 0,4 a 0,9 , utilizando correção gama com valor 1 . Os parâmetros para ambas as técnicas são definidos empiricamente em vista de quais valores apresentam melhores resultados nos testes.

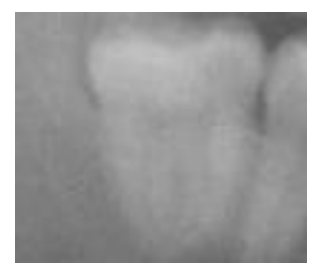

(a)

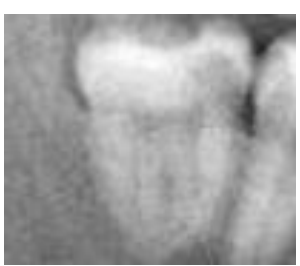

(b)

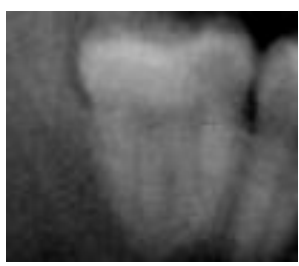

(c)

Figura 3. Pré-processamento. (a) imagem original. (b) imagem com contraste morfológico; (c) imagem com contraste por ajuste de tons.

A Figura 3 apresenta a aplicação dessas operações juntamente com o filtro gaussiano. A Figura 3(a) apresenta a imagem original, a Figura 3(b) com aplicação do contraste morfológico e a Figura 3(c) com aplicação do contraste por ajuste. É possível observar que após as técnicas utilizadas a região referente ao dente fica visivelmente mais destacada em comparação com a imagem original.

\subsection{Classificação}

As imagens foram redimensionadas para a resolução de 256x256, visando padronizar o tamanho de todas as imagens, e passaram por uma normalização de pixels antes de serem aplicadas nas CNNs. Essa normalização consiste em dividir todos os pixels pelo de maior valor, garantindo que todos possuam valores no intervalo de 0 a 1 . Nos experimentos, são utilizadas duas arquiteturas, uma que apresenta redução da quantidade de filtros ao longo das camadas de convolução e outra que apresenta crescimento. Elas serão referenciadas como CNN1 e CNN2, respectivamente, ao longo do artigo. O uso dessas arquiteturas é proposto para investigar a influência no resultado de detecção, realizando redução e aumento do número de filtros ao longo das camadas. Nas imagens das estruturas das CNNs a seguir, as barras das camadas convolucionais possuem tamanho ilustrando a quantidade de filtros aplicados. A arquitetura da CNN1 é apresentada na Figura 4.

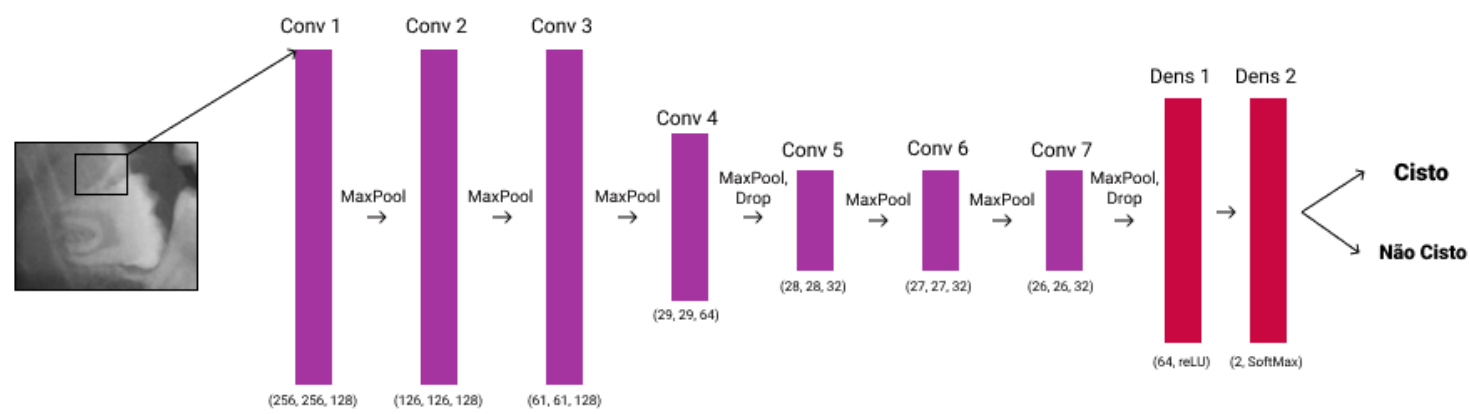

Figura 4. Arquitetura da CNN1. 
Nas camadas de convolução a CNN1 utiliza strides com valor 1, padding nas entradas e quantidade de filtros de 128, 64 e 32, cada um utilizando janelas de tamanho $3 \times 3$. As camadas de ativação após as convoluções utilizam função ReLu (Rectified Linear Unit), uma função frequentemente utilizada em problemas de classificação e que tem apresentado bons resultados em aplicações convolucionais [LeCun et al. 2015].

Após cada convolução, são utilizadas camadas de pooling com a técnica de MaxPooling e tamanho $2 \times 2$. Essa técnica mantém apenas os maiores valores dentro de uma janela amostral do vetor resultante da convolução, com isso ocorre uma redução de dimensionalidade. A arquitetura utiliza também a técnica de dropout de 0,5 após as camadas de convolução 4 e 7 . Finalizando as camadas convolucionais, ela utiliza duas camadas totalmente conectadas, uma com 64 neurônios utilizando função de ativação ReLu e outra com 2 (equivalente ao número de classes), utilizando a função de ativação Softmax. A Figura 5 apresenta a arquitetura da CNN2.

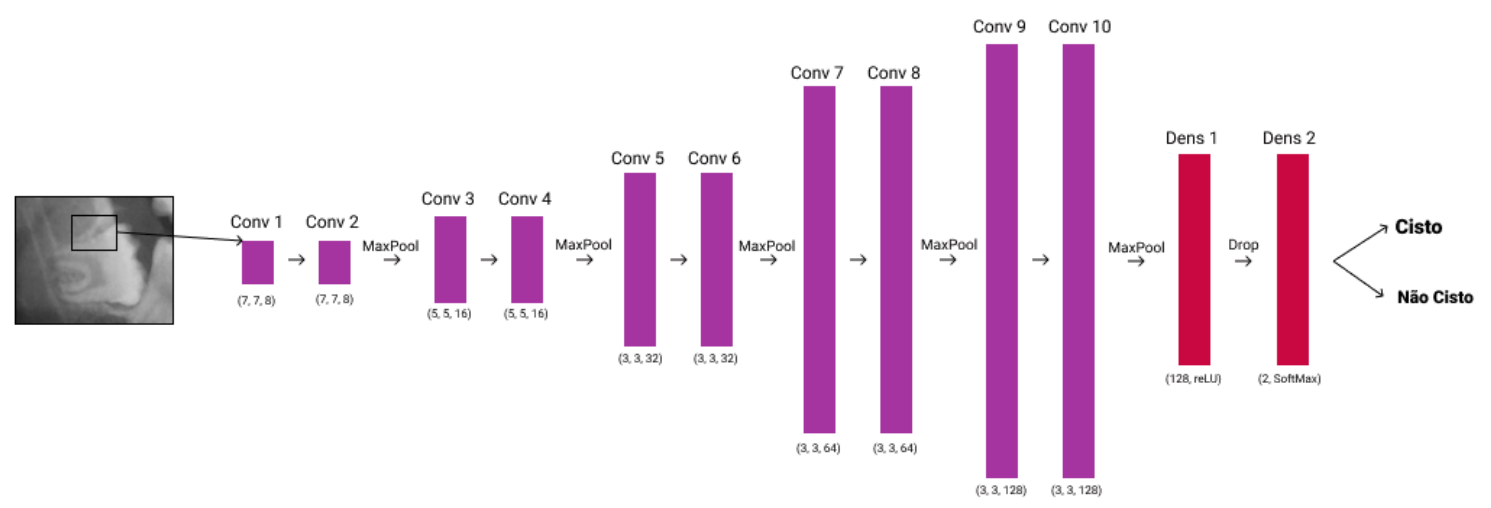

Figura 5. Arquitetura da CNN2.

Nas convoluções a CNN2 utiliza camadas duplas para cada quantidade de filtros, com quantidades de 8, 16, 32, 64 e 128 filtros, aplicando também padding nas entradas de cada convolução e strides com valor 1 . Os tamanhos das janelas utilizadas nos filtros são de $3 \times 3,5 \times 5$ e $7 \times 7$. Nela, todas as camadas de ativação utilizam função ReLu. As camadas de pooling são aplicadas após as duplas camadas de convolução, também utilizando MaxPooling com tamanho 2x2.

Após as convoluções ela utiliza duas camadas totalmente conectadas, com 128 e 2 neurônios (número de classes). A primeira utilizando a função de ativação ReLu e a segunda a SoftMax. Entre essas camadas é aplicada a técnica de dropout com 0,2.

Os parâmetros de treinamento utilizados são os mesmos para ambas as redes, com o otimizador Adaptive Moment Estimation (Adam), com taxa de aprendizado de 0,0001. As redes são treinadas com 500 épocas, utilizando entropia cruzada como função de perda e o parâmetro batch com tamanho 3 .

\subsection{Realização do experimento}

Nessa etapa, a metodologia é aplicada na técnica de validação cruzada conhecida como $k$-fold. Essa é uma técnica clássica de validação de modelos de predição, que consiste em dividir as amostras em $k$ grupos e utilizar $k-1$ das amostras para treino e as restantes para teste, fazendo esse processo $k$ vezes. No entanto, para um treino efetivo de 
uma CNN é necessário um grande número de amostras. Dessa forma, a pesquisa também utiliza a técnica de expansão de imagens do banco.

Essa técnica de expansão consiste em aplicar transformações nas imagens com objetivo de obter mais amostras, tais como translações nos eixos, rotação, perspectiva e combinações entre elas. A expansão é aplicada individualmente nos grupos de treino e teste após a separação do $k$-fold. Dessa forma, é garantido que as amostras participantes do grupo de teste em cada iteração não tenham sido vistas durante o treino. São geradas um total de 17.010 imagens e o $k$-fold é aplicado com $k=10$.

\section{Resultados e discussão}

A análise dos resultados de cada metodologia é dada pela média das métricas obtidas de cada partição do $k$-fold, sendo comparada para cada arquitetura a classificação das imagens com os dois métodos de pré-processamento e a aplicação na imagem original. Os resultados das arquiteturas CNN1 e CNN2 são observados nas Tabela 1 e Tabela 2, respectivamente.

Tabela 1. Média das métricas obtidas da arquitetura CNN1.

\begin{tabular}{cccc}
\hline & Sem pré-processamento & $\begin{array}{c}\text { Contraste } \\
\text { morfológico }\end{array}$ & $\begin{array}{c}\text { Contraste por } \\
\text { ajuste }\end{array}$ \\
\hline Acurácia & 0,7557 & 0,7870 & 0,7046 \\
\hline Precisão & 0,8161 & 0,7930 & 0,6642 \\
\hline Sensibilidade & 0,6486 & 0,7724 & 0,8229 \\
\hline F1-score & 0,7171 & 0,7727 & 0,7262 \\
\hline
\end{tabular}

Percebe-se que a CNN1 apresentou melhores resultados que a CNN2 para a aplicação direta na imagem original, enquanto para os dois testes com pré-processamento a arquitetura da CNN2 obteve maiores valores para quase todas as métricas, com exceção da sensibilidade. Percebe-se também que ambos os pré-processamentos elevaram os resultados da CNN2 em todas as métricas, enquanto para a CNN1 apenas a sensibilidade teve um aumento considerável.

Tabela 2. Média das métricas obtidas da arquitetura CNN2.

\begin{tabular}{cccc}
\hline & Sem pré-processamento & $\begin{array}{c}\text { Contraste } \\
\text { morfológico }\end{array}$ & $\begin{array}{c}\text { Contraste por } \\
\text { ajuste }\end{array}$ \\
\hline Acurácia & 0,6530 & 0,8702 & 0,7880 \\
\hline Precisão & 0,5760 & 0,9346 & 0,7620 \\
\hline Sensibilidade & 0,7441 & 0,7718 & 0,8064 \\
\hline F1-score & 0,6354 & 0,8426 & 0,7655 \\
\hline
\end{tabular}

A CNN1 aplicada com o contraste morfológico resulta no aumento de quase todas as métricas, reduzindo apenas a precisão de 0,81 para 0,79 . O mesmo processo utilizando 
o contraste por ajuste resulta na redução das métricas de acurácia e precisão e no aumento da sensibilidade e F1-score.

Aplicando a CNN2 com o contraste morfológico, observa-se também a influência positiva desse contraste na capacidade de indicar corretamente um cisto, aumentando a precisão de 0,57 para 0,93 . O mesmo processo utilizando o contraste por ajuste também resulta no aumento significativo das métricas, mas com variação menos acentuada que o anterior.

A melhor performance apresentada é dada pela CNN2 utilizando o contraste morfológico. Embora essa metodologia tenha exibido uma sensibilidade levemente inferior do que os outros testes com pré-processamento, é o único teste que obteve acurácia e F1-score acima de 0,8 e uma precisão acima de 0,9. O contraste morfológico apresenta métricas superiores devido sua caracterítica de fornecer maior discriminação visual entre as estruturas e permitir melhor identificação das regiões císticas. O menor desempenho foi reconhecido nos resultados do experimento com a CNN2 sem préprocessamento, que mesmo tendo uma sensibilidade superior a da CNN1 sem préprocessamento, atingiu os menores valores para as outras métricas em comparação com todos os outros testes.

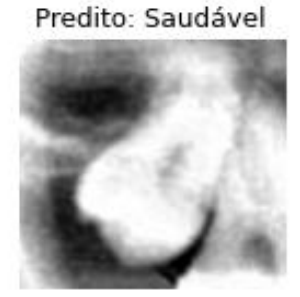

Diagnóstico: Saudável

Predito: Saudável

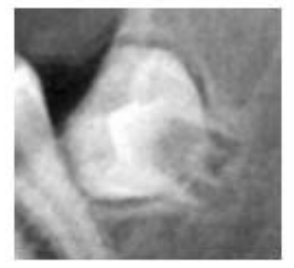

Diagnóstico: Cisto

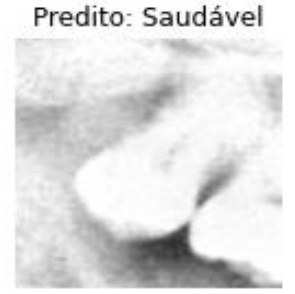

Diagnóstico: Saudável

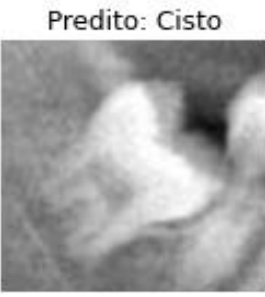

Diagnóstico: Cisto

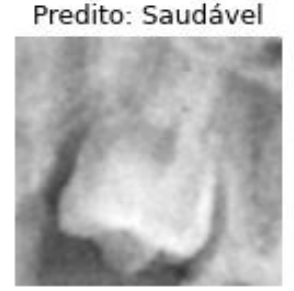

Diagnóstico: Saudável

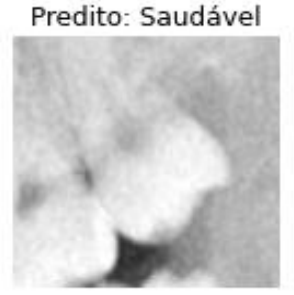

Diagnóstico: Saudável

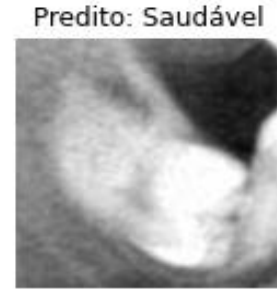

Diagnóstico: Cisto

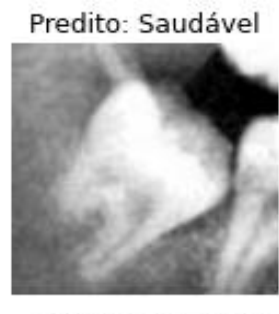

Diagnóstico: Cisto

Figure 6. Resultados da CNN2 com contraste morfológico.

Alguns resultados do melhor experimento são apresentados na Figura 6. É possível identificar que a informação predita é o valor obtido pela metodologia, e o diagnóstico é o valor informado pelos especialistas. Nesse grupo, observa-se a presença de três imagens com predições incorretas e cinco corretamente classificadas. Além disso, percebe-se que apesar da complexidade e do alto teor de ruídos das imagens, a metodologia experimentada com base em $\mathrm{CNN}$ e pré-processamento com filtro gaussiano e contraste morfológico resultou em uma detecção considerável, apresentando potencial para auxílio ao diagnóstico. 


\section{Conclusão}

Neste trabalho foi utilizado duas redes neurais convolucionais, uma com crescimento do número de filtros ao longo das camadas e outra com decrescimento. Essas redes são implementadas, treinadas e testadas em conjuntos com técnicas de pré-processamentos para classificar imagens de dentes sisos como saudáveis ou com presença de cisto.

A pesquisa cumpre o objetivo, desenvolvendo uma metodologia que identifique a presença de cistos nas imagens utilizadas e investigando a influência das técnicas de préprocessamento. A proposta alcançou melhores resultados através da arquitetura com crescimento do número de filtros ao longo das camadas. Além disso, também foi concluído que as técnicas de pré-processamento influenciaram positivamente os resultados das redes, sendo o contraste morfológico o que obteve melhores métricas.

Diante dos resultados obtidos a pesquisa se mostrou promissora para o auxílio no diagnóstico de radiografias de dentes sisos, classificando em saudáveis ou com presença de cisto. Como trabalhos futuros pretende-se aplicar essa metodologia em conjunto a um sistema de segmentação de dentes sisos em imagens de radiografias panorâmicas. Além disso, pretende-se também avaliar o uso de mais técnicas de pré-processamento e outras arquiteturas de Redes Neurais Convolucionais.

\section{References}

Zanetti, L. S. S., Marano, R. R., Bianchi, P. R., Garcia Junior, I. R. and de Barros, A. P. (2009). Transformação Cística como Consequência de Terceiro Molar Inferior Incluso. Revista Portuguesa de Estomatologia, Medicina Dentária e Cirurgia Maxilofacial, 50(1): 19 -23, doi: 10.1016/S1646-2890(09)70012-3.

Slootweg, P. J. (1987). Carcinoma arising from reduced enamel epithelium. Journal of Oral Pathology, 16(10): 479 - 482, doi: 10.1111/j.1600-0714.1987.tb00676.x.

Ulaganathan, G., Banumathi, A., Amutha, J. J. and Jeevani Selvabala, A. (2012). Dental Cyst Delineation Using Live Wire Algorithm. In 2012 International Conference on Machine Vision and Image Processing (MVIP), pages 129 - 132, doi: 10.1109/MVIP.2012.6428777.

Salehinejad, J., Saghafi, S. and Ghazi, N. (2013). Glandular odontogenic cyst associated with an impacted tooth. Journal of Dental Materials and Techniques, 2: 99 - 103, doi: 10.22038/JDMT.2013.1053.

Patil, S. (2013). Prevalence and type of pathological conditions associated with unerupted and retained third molars in the western Indian population. Journal of CranioMaxillofacial Surgery, 2(1): $3-4$.

Birdal, R. G., Gumus, E., Sertbas, A. and Birdal, I. S. (2016). Automated lesion detection in panoramic dental radiographs. Oral Radiology, 32(1): 111 - 118, doi: 10.1007/s11282-015-0222-8.

Banar, N., Bertels, J., Laurent, F., Boedi, R. M., Tobel, J. D., Thevissen, P. and Vandermeulen, P. (2020). Towards fully automated third molar development staging in panoramic radiographs. International Journal of Legal Medicine, 134(1): 18311841, doi: 10.1007\%2Fs00414-020-02283-3.

Divya, K. V., Jatti, A., Joshi, R. and Krishna, S. D. (2017). Characterization of dental pathologies using digital panoramic X-ray images based on texture analysis. In 39th Annual International Conference of the IEEE Engineering in Medicine and Biology Society (EMBC), pages $11-15$, doi: 10.1109/EMBC.2017.8036894. 
Lee, J. H., Kim, D. H. and Jeong, S. N. (2020). Diagnosis of cystic lesions using panoramic and cone beam computed tomographic images based on deep learning neural network. Oral diseases, 26(1): 152 - 158, doi: 10.1111/odi.13223.

Devi, R. K., Banumathi, A., Sangavi, G. and Dawood, M. S. (2020). A Novel Region Based Thresholding for Dental Cyst Extraction in Digital Dental X-Ray Images. New Trends in Computational Vision and Bio-inspired Computing, pages 1633 - 1640, doi: 10.1007/978-3-030-41862-5_167.

Kwon, O., Yong, T. H., Kang, S. R., Kim, J. E., Huh, K. H., Heo, M. S., Lee, S. S., Choi, S. C. and Yi, W. J. (2020). Automatic diagnosis for cysts and tumors of both jaws on panoramic radiographs using a deep convolution neural network. Dentomaxillofacial Radiology, 49(8): 20200185, doi: 10.1259/dmfr.20200185.

Birdal, R. G., Gumus, E., Sertbas, A. and Birdal, I. S. (2015). Automated lesion detection in panoramic dental radiographs. Oral Radiology, 32(2): 111 - 118, doi: 10.1007/s11282-015-0222-8.

Goodfellow, I., Bengio, Y. and Courville, A. (2016). Deep learning. [S.1.]: MIT press.

LeCun, Y., Bottou, L., Bengio, Y. and Haffner, P. (1998). Gradient-based learning applied to document recognition. In Proceedings of the IEEE, 86(11): 2278 - 2324.

Gu, J., Wang, Z., Kuen, J., Ma, L., Shahroudy, A., Shuai, B., Liu, T., Wang, X. Wang, G., Cai, J. and Chen, T. (2017). Recent advances in convolutional neural networks. Pattern Recognition, 77(1): $354 \quad-\quad 377, \quad$ doi: https://doi.org/10.1016/j.patcog.2017.10.013. 